\title{
High resolution examination of the role of sleep disturbance in predicting functioning and psychotic symptoms in schizophrenia: \\ DOI:
}

10.1037/abn0000180

\section{Document Version \\ Accepted author manuscript}

Link to publication record in Manchester Research Explorer

Citation for published version (APA):

Mulligan, L., Haddock, G., Emsley, R., Neil, S. T., \& Kyle, S. (2016). High resolution examination of the role of sleep disturbance in predicting functioning and psychotic symptoms in schizophrenia: A novel experience sampling study. Journal of Abnormal Psychology, 125(6), 788-797. https://doi.org/10.1037/abn0000180

\section{Published in:}

Journal of Abnormal Psychology

\section{Citing this paper}

Please note that where the full-text provided on Manchester Research Explorer is the Author Accepted Manuscript or Proof version this may differ from the final Published version. If citing, it is advised that you check and use the publisher's definitive version.

\section{General rights}

Copyright and moral rights for the publications made accessible in the Research Explorer are retained by the authors and/or other copyright owners and it is a condition of accessing publications that users recognise and abide by the legal requirements associated with these rights.

\section{Takedown policy}

If you believe that this document breaches copyright please refer to the University of Manchester's Takedown Procedures [http://man.ac.uk/04Y6Bo] or contact uml.scholarlycommunications@manchester.ac.uk providing relevant details, so we can investigate your claim.

\section{OPEN ACCESS}


High-resolution examination of the relationship between sleep disturbance, functioning and psychotic symptom severity in schizophrenia: a novel experience sampling study

\author{
Lee D. Mulligan ${ }^{\mathrm{a}^{*}}$ \\ Gillian Haddock ${ }^{\mathrm{a}}$ \\ Richard Emsley ${ }^{\mathrm{b}}$ \\ Sandra T. Neil ${ }^{\mathrm{c}}$ \\ Simon D. Kyle
}

${ }^{\mathrm{a}}$ University of Manchester, School of Psychological Sciences, Zochonis Building, Second Floor, Brunswick Street, Manchester, M13 9PL

${ }^{\mathrm{b}}$ University of Manchester, Centre for Biostatistics, Institute of Population Health, Jean McFarlane Building, Oxford Road, Manchester, M13 9PL

${ }^{\mathrm{c}}$ Greater Manchester West Mental Health NHS Foundation Trust, Cromwell House Community Mental Health Team, Manchester, M30 0QT

${ }^{\mathrm{d}}$ University of Oxford, Sleep and Circadian Neuroscience Institute, Nuffield Department of Clinical Neurosciences, Level 6, West Wing, John Radcliffe Hospital, Oxford, OX3 9DU

${ }^{*}$ Corresponding Author: School of Psychological Sciences, University of Manchester, $2^{\text {nd }}$ Floor Zochonis Building, Brunswick Street, Manchester, M13 9PL

UK

Telephone: +44 (0)1613060400

Email address: lee.mulligan@nhs.net 


\begin{abstract}
Sleep disturbance is common in schizophrenia but its role in predicting functioning and psychotic symptoms has yet to be rigorously examined. The purpose of this study was to conduct a prospective, high-resolution examination of the relationship between nightly sleep and next-day functioning and psychotic symptoms in people with a diagnosis of schizophrenia. Experience sampling methodology was integrated with actigraphy and sleep diaries across seven days in twenty-two patients with a diagnosis of schizophrenia. Momentary assessments of mood, psychotic symptoms and functioning were gathered at five points each day following pseudo-random schedules. Multilevel modelling was performed to evaluate the links between variables. Both objective and subjective sleep disturbance predicted reduced next-day functioning, which remained significant after controlling for psychotic symptom severity. Increased sleep fragmentation and reduced subjective and objective sleep efficiency predicted greater next-day auditory hallucinations, whereas increased objective sleep fragmentation and reduced subjective sleep quality predicted greater paranoia and delusions of control. Negative affect on awakening mediated a proportion of these relationships (range: 17.9\% - 57.3\%). For the first time, we show that sleep disturbance is a predictor of next-day impaired functioning and psychotic symptom severity in people with a diagnosis of schizophrenia. Interventions targeting sleep may therefore have the potential to enhance functional and symptomatic recovery in those experiencing psychosis, both directly and indirectly.
\end{abstract}

Keywords: schizophrenia, psychosis, sleep, insomnia, functioning

General Scientific Summary

Sleep problems are common in people with a diagnosis of schizophrenia. This study shows that objective and subjective sleep disturbance significantly predicts next-day impaired functioning and greater psychotic symptoms, which may be partially explained by negative affect. 
Sleep disturbance is considered an important transdiagnostic factor, implicated in the development and maintenance of a range of psychiatric disorders (Harvey, 2008). Estimates indicate that between $30-80 \%$ of patients with a schizophrenia diagnosis report sleep continuity difficulties (Cohrs, 2008), which can be observed in the prodromal (Davies, Haddock, Yung, Mulligan \& Kyle, 2016) acute (Kupfer, 1970) and residual (Waters et al., 2011) phases of the disorder. Sleep problems are associated with adverse clinical outcomes, including elevated rates of depression and completed suicide (Palmese et al., 2011; Pompili et al., 2009). Studies have also demonstrated that sleep disturbance is associated with increased psychosis severity, when measured subjectively (Xiang et al., 2009) or objectively (Waters et al., 2011). For instance, there is evidence to suggest a positive relationship between insomnia and severity of both delusions (Myers, Startup, \& Freeman, 2011) and hallucinations (Reich, Weiss, Coble, McPartland, \& Kupfer, 1975). However, a recent systematic review of the relationship between sleep disturbance and psychosis highlighted a dearth of well-controlled, longitudinal studies examining sleep parameters and individual psychotic symptoms, especially in people with a diagnosis of schizophrenia (Reeve, Sheaves \& Freeman, 2015). Examination of putative mechanisms connecting sleep disturbance to psychosis is also limited but could plausibly relate to reasoning biases, altered sensory gating, disturbance in neurotransmitter regulation, and emotional distress (anxiety, depression).

Moreover, comparatively little research has investigated the effects of sleep disturbance on functioning in people with a diagnosis of schizophrenia. This is despite service users emphasising the importance of functioning in their recovery (Wood, Price, Morrison, \& Haddock, 2011). Indeed, research has shown that sleep disturbance is associated with impaired quality of life (QoL) and coping in people experiencing psychosis (Ritsner, Kurs, Ponizovsky, \& Hadjez, 2004; Hofstetter, Lysaker, \& Mayeda, 2005). Neuropsychological findings also demonstrate that sleep disturbance contributes to impaired performance on tasks of sustained 
attention (Forest et al., 2007), executive functioning (Keshavan, Cashmere, Miewald, \& Yeragani, 2004) and IQ (Manoach et al., 2014). However, whether these performance deficits approximate daily functioning remains unclear.

To date, studies examining the relationship between sleep, functioning and psychosis severity have been 1) cross-sectional in nature; 2) have used either subjective (e.g., questionnaires) or objective (e.g., polysomnography) measures of sleep in isolation, despite guidelines advocating the measurement of both and the potential for discrepancy between subjective and objective reports (Buysse, Ancoli-Israel, Edinger, Lichstein \& Morin, 2006); and 3) have employed low-resolution or proxy measures to assess psychotic symptoms and daytime functioning. While this work has been informative, low-resolution and cross-sectional studies are vulnerable to recall bias, are limited in depth and range of measurement, and are unable to examine relationships on a micro-scale. This is particularly important as sleep is multi-dimensional in nature and can fluctuate across nights, with corresponding next-day consequences. The temporal relationship between nightly sleep and next-day functioning and psychotic symptoms has yet to be systematically investigated, despite clear clinical implications. Studies employing high-resolution measurement of sleep and next-day variables over time would help to clarify the dynamic relationship between different sleep dimensions, psychotic symptoms and functioning, and would provide opportunities to examine mediating relationships.

In the present study, we addressed these important questions through the novel integration of experience sampling methodology - to assess real-time psychotic symptoms and functioning - with objective and subjective measures of nightly sleep. We hypothesised that greater objective and subjective sleep disturbance would predict impaired functioning and greater symptom severity, across the following day. We also examined the mediating impact of negative affect on these relationships, since 1) sleep disturbance, functioning and symptoms 
are all associated with negative affect, 2) preliminary studies find evidence for partial mediation of the link between insomnia severity and paranoia via negative affect (Freeman, Pugh, Vorontsova \& Southgate, 2009; Myers et al., 2011) and 3) cognitive models of psychosis identify negative affect as an important contributory and maintaining factor in both paranoia and auditory hallucinations (Waters et al., 2012; Freeman \& Garety, 2003). Moreover, increased negative affect has been reliably demonstrated after experimental sleep restriction and fragmentation in non-clinical studies (Finan, Quartana \& Smith, 2015), representing a potential pathway through which both impaired functioning and symptom severity may be potentiated by sleep disturbance in vulnerable populations.

\section{Method}

Participants

The study was reviewed and approved by the North West 7 UK NHS Research Ethics Committee (REC ref: 14/NW/0248) and by local research and development offices. Following approval, all participants were recruited from four mental health trusts across the North West of England.

All participants held or met criteria for a diagnosis of schizophrenia, schizophreniform disorder, schizoaffective disorder, delusional disorder, psychotic disorder not otherwise specified, or non-affective psychosis, in accordance with the International Classification of Diseases-10 (ICD-10; World Health Organisation, 1992) and Diagnostic and Statistical Manual for Mental Disorders (DSM-V; American Psychiatric Association, 2013), as confirmed by a psychiatrist in their mental health care. All participants were required to score $>=10$ on the Insomnia Severity Index (Bastien, Vallières, \& Morin, 2001), were currently receiving support 
from mental health services, had a command of the English language sufficient to complete assessments and were able to provide informed consent for participation.

Participants were excluded if they: (i) had a primary organic disorder; (ii) had a learning disability; (iii) were currently receiving inpatient care; (iv) were experiencing an acute episode of psychosis; (v) met criteria for sleep disorder other than insomnia, as assessed by a screening measure administered before participation.

Measures

All screening and baseline measures were completed by the lead author (LDM),

Screening measures

Two screening measures were administered to confirm the eligibility of all participants. Both were administered by telephone.

Insomnia Severity Index (ISI). The seven-item ISI (Bastien et al., 2001) was used to assess the severity and impact of insomnia. Each item is measured on a five-point Likert scale $(0=$ no problem; $4=$ very severe problem $)$ and total scores range from 0 to 28 . A cut-off score of 10 was used to determine eligibility, as this has been judged as optimal for identifying insomnia with the highest sensitivity and specificity (Morin, Belleville, Bélanger, \& Ivers, 2011). The psychometric properties of the ISI have been widely reported (Yang, Morin, Schaefer, \& Wallenstein, 2009) and the measure has been used in studies including psychosis populations (Myers et al., 2011).

Brief Screen for Sleep Disorders. A five-item screening tool (Wilson et al., 2010) was used to assess for sleep disorder pathology, including narcolepsy, sleep breathing disorder, periodic limb movement syndrome or restless leg syndrome, circadian rhythm sleep disorder, 
and parasomnia. Participants were excluded if they endorsed any screening item, in addition to at least one branching item.

Baseline measures

Six baseline measures were administered before the ESM phase to ascertain retrospective measures of psychosis, functioning and substance use. Primarily, these were chosen to characterise the sample, but also to provide opportunities to control for confounders of the relationship between sleep, functioning and psychotic symptoms.

Symptom measures

Positive and Negative Syndrome Scale (PANSS). The thirty-item PANSS (Kay, Fiszbein, \& Opler, 1987) was conducted to measure overall symptom severity. All items are rated on ordinal scales $(1=$ absent, $7=$ extreme $)$ and total scores range from 30 to 210 . The PANSS can be subdivided into positive, negative and general psychopathology scores and both the total and its subscales have high reliability and validity (Kay, Opler \& Lindenmayer, 1988).

Psychotic Symptom Rating Scale (PSYRATS). The PSYRATS (Haddock, McCarron, Tarrier, \& Faragher, 1999) was used to measure multidimensional experiences of auditory hallucinations (eleven items) and delusions (six items); specifically, emotional and physical characteristics plus cognitive interpretations. All items are rated on ordinal scales (0-4) and total scores range from 0 to 44 (auditory hallucinations) and 0 to 24 (delusions). The PSYRATS has been widely validated and has high reliability (Haddock et al., 1999).

Calgary Depression Scale (CDS). The nine-item CDS (Addington, Addington, \& Maticka-Tyndale, 1993) was used to measure depression severity in the context of 
schizophrenia. Each item is scored on a four-point Likert scale $(0=$ absent, $3=$ severe $)$ and total scores range from 0 to 27 . The CDS has proven reliability and validity in populations with a diagnosis of schizophrenia (Addington et al., 1993).

Substance use measures

Drug Abuse Screening Test (DAST-10). The ten-item DAST (Skinner, 1982) was used to quantify severity of drug use. Each item is scored on a dichotomous scale $(0=$ no; $1=$ yes $)$ and total scores range from 0 to 10 . The reliability and validity of the DAST-10 has been demonstrated in samples experiencing psychosis (Cassidy, Schmitz, \& Malla, 2008).

Alcohol Use Disorders Identification Test (AUDIT-10). The ten-item AUDIT (Saunders, Aasland, Babor, De la Fuente, \& Grant, 1993) was used to measure alcohol consumption and alcohol-related difficulties. All items are scored on ordinal scales (0-4) and total scores range from 0 to 40 . The AUDIT is reliable and valid for use with people experiencing psychosis (Dawe, Seinen, \& Kavanagh, 2000).

Functioning measures

Personal and Social Performance Scale (PSP). The PSP (Morosini, Magliano, Brambilla, Ugolini, \& Pioli, 2000) was used to measure functioning across four domains, including socially useful activities, personal and social relationships, self-care and aggression. Total scores range from 0 to 100 , with higher scores indicating greater functioning. The PSP is valid and reliable for use in samples with a diagnosis of schizophrenia (Kawata \& Revicki, 2008). 
Daily sleep measures

Actigraphy. Objective measurement of sleep-wake patterns was conducted using wrist actigraphy (CamNtech Patient Recorded Outcome-Diary, Cambridge) over a period of six days and nights, utilising an epoch of thirty seconds. Actigraphy provides a recording of continuous motor activity and has been validated for use within psychosis populations (Tahmasian, Khazaie, Golshani, \& Avis, 2013). All participants were instructed to wear the actigraph on the non-dominant wrist for the entire study period. A sleep diary was used to define time-inbed periods for actigraph monitoring, with automated algorithms generating the following nightly sleep variables:

- Sleep Efficiency (SE): Actual sleep time expressed as a percentage of time in bed.

- Sleep Fragmentation (SF): An index of time spent mobile during the night.

- Total Sleep Time (TST): The total time spent in sleep according to the epoch-by-epoch wake/sleep categorisation.

Consensus Sleep Diary (CSD). The CSD (Carney et al., 2012) was completed by participants each morning to measure subjective sleep parameters. Three sleep variables were extracted: SE, TST and sleep quality (SQ). Approximate times in and out of bed were used to define sleep periods for actigraphic investigation. The CSD was developed by sleep experts and has been credited as a gold-standard sleep diary (Carney et al., 2012).

ESM measures

Item development

Thirteen emotion and psychotic symptom items were generated based on previous studies utilising ESM with individuals experiencing psychosis (Hartley, Haddock, 
Vasconcelos, Emsley, \& Barrowclough, 2014). To ensure their relevance, eleven functioning items were developed in collaboration with individuals with lived experience of psychosis. A focus group was conducted with three people to explore their experience of sleep and daily functioning. Themes derived from this focus group were used to generate items capturing people's experience of daily functioning, following poor sleep. All items were refined to ensure sensitivity to momentary changes (Palmier-Claus et al., 2011) and wordings were checked by focus group members and revised based on their feedback, to maximise relevance. Reliability analysis (Cronbach's alpha) was computed to ensure scale adequacy.

\section{Emotion items}

At every assessment point, participants were instructed to rate the extent to which they felt sad, cheerful, anxious, happy, irritable, relaxed, sleepy and energetic immediately prior to the entry point. These were chosen based on previous ESM studies to provide subscale measures of negative affect (NA) and positive affect (PA) (Palmier-Claus, Dunn, \& Lewis, 2012). All items were rated on seven-point Likert scales ( $1=$ not at all, $7=$ very much so). The NA (sad, anxious, irritable) and PA (happy, cheerful, relaxed, sleepy and energetic) subscales had good internal consistency ( $\alpha=.808$ and $\alpha=.797$, respectively). Mean NA scores were used in the analyses.

\section{Psychotic symptom items}

At every assessment point, participants were instructed to rate the extent to which they were experiencing psychotic symptoms, immediately prior to the entry point. Psychosis items included: hearing voices, seeing things, feeling that someone or something may cause me harm, feeling that I have special powers or abilities and feeling that my thoughts are being influenced 
or controlled. These were chosen to provide measures of hallucinations (auditory, visual) and delusions (persecutory, grandiose, control); however, these scales did not demonstrate high levels of internal consistency ( $\alpha=.498$ and $\alpha=.677$, respectively). Therefore, individual scores were used in the analyses. Analysis of frequencies suggested that visual hallucinations and grandiosity were not sufficiently endorsed across the study period [i.e. less than $20 \%$ of entry points were rated greater than 1 ("not at all”)]. Subsequently, only scores for auditory hallucinations, paranoia and delusions of control were included in the analyses.

\section{Functioning items}

At every assessment point, participants were instructed to rate their ability to function, since the last entry. Items included: I have found it difficult to motivate myself, remember things, get things done; I have been able to concentrate, think clearly, make decisions, look after myself; I have felt able to leave the house, participate in enjoyable activities, spend time with loved ones and talk to people. Items were either forward or reversed scored, with higher scores indicating greater functioning. The total functioning scale was used in the analyses, which had high internal consistency $(\alpha=.905)$.

\section{ESM and sampling procedure}

All ESM items were uploaded onto the CamNtech PRO-Diary (CamNtech Ltd, Cambridge), programmed with the integrated function of administering and recording momentary assessments, alongside actigraphy. Participants were prompted to complete entries at regular intervals using the slider and select keys on the watch display, in response to an electronic alarm. The prompts were delivered five times per day over a period of up to seven days. The quasi-random ESM schedule was personalised for each participant based on their 
self-reported, habitual times of sleep onset and offset. The first and last prompts of the schedule were delivered within approximately one hour of sleep offset and onset for each participant. After dividing the number of minutes between the first and last prompt by three, the remaining prompts were scheduled so that each occurred periodically across each day, for each participant. This sampling strategy was employed to maximise adherence to the ESM procedure (Kimhy, Myin-Germeys, Palmier-Claus, \& Swendsen, 2012).

Procedure

A visual depiction of the study procedure can be found in Supplementary Figure 1.

Recruitment / screening phase

All participants were recruited from community mental health teams and early intervention in psychosis services across the North West of England. All participants were identified and approached by a member of their clinical team to participate in this study. All verbally consenting participants were screened for eligibility by telephone, prior to completion of the baseline assessments. Participants who did not meet eligibility criteria were informed of the reasons for this and were debriefed. For all eligible participants, approximate times of sleep onset and offset were elicited to ensure all ESM schedules could be personalised for each participant.

Baseline phase

All eligible participants were visited in their homes to ascertain written consent for participation. Once received, baseline measures were administered. After completion, all 
participants were briefed about the sleep / ESM phase. Time was taken to familiarise participants with the PRO-Diary, the ESM question and answer format and the personalised schedule. The researcher also familiarised participants with the sleep diary and provided instructions and contact details for use in case of difficulty.

Sleep / ESM phase

On the first day of the sleep / ESM phase, each participant was contacted by the researcher to confirm the functionality of the PRO-Diary and ensure that all entries were being completed correctly. This also enabled the researcher to further emphasise the voluntary nature of participation and the rights of participants to withdraw from the study, if they so wished, at any time.

Post-sleep / ESM phase

Following the ESM phase, all participants were debriefed and both the PRO-Diary and sleep diaries were returned.

Analysis

All analyses were performed on STATA version 12.1. All participant-level and daylevel data were assessed for normality via histogram inspection and analysis of skewness and kurtosis statistics. The sleep fragmentation and psychosis symptom day-level variables were positively skewed and subsequent analyses used non-parametric bootstrapping with 200 
replications to account for non-normality (Mooney, Duval, \& Duval, 1993). ESM measures typically have a 3-level hierarchical structure (entry points, nested within days, nested within participants), which violates the assumption of independent observations; therefore, multilevel modelling was performed to account for the clustering in outcomes. As sleep variables can only be observed once per day, we used a summary of beep level measures across the following day.

The analysis strategy involved the computation of total and direct effects of day-level variables. For all analyses, participant number was included as a random effect to control for the nested structure of ESM data and no predictors were centred. Firstly, a multilevel regression analysis utilising a random effects model ('XTREG') with maximum likelihood estimation ('MLE') was used to explore whether objective and subjective sleep parameters predicted next-day functioning and psychotic symptoms, whilst adjusting for PANSS score. Secondly, to test the effect of morning NA as a potential mediator of the relationship between sleep parameters and next-day functioning and symptoms, we used the differences in coefficients approach (Bolger \& Laurenceau, 2013). This involved first examining whether sleep variables predicted morning NA and then comparing the coefficients between the total effect of sleep parameters on next-day variables (model one) and the direct effect of sleep parameters on next-day variables, when morning NA was added as mediator (model two). The difference between these models represents the indirect effect. Morning NA was chosen specifically, to ensure temporal separation between mediator (affect) and dependent variables (psychotic symptoms and functioning). 


\section{Results}

Twenty-four people consented to participate, of which twenty-two met eligibility criteria following screening. Two participants were excluded on the basis of meeting criteria for circadian rhythm disturbances. A full list of demographic and clinical characteristics of the sample is summarised in Table 1. All participants were taking antipsychotic medication, sixteen in augmentation with antidepressants. Antipsychotic medications included amisulpride, aripiprazole, clozapine, olanzapine, quetiapine and risperidone, with doses ranging from 10mg to $800 \mathrm{mg}$. Antidepressant medications included mirtazapine, sertraline and venlafaxine, with doses ranging from $40 \mathrm{mg}$ to $400 \mathrm{mg}$.

The sample was chronic in presentation with a mean duration since diagnosis of twelve years $($ S.D. $=6.2)$. The majority of participants held a diagnosis of schizophrenia $(n=13)$ or non-affective psychosis $(n=8)$, while one participant held a diagnosis of schizoaffective disorder. In terms of baseline measures, the sample was characterised by mild overall psychotic symptom severity. Mean ISI scores indicated that participants were experiencing insomnia of moderate severity. According to cut-off scores, 16 participants met criteria for major depression. Levels of alcohol and drug use were low.

Retention and Adherence

All twenty-two participants completed the full ESM schedule and completed more entries than the traditionally accepted one-third of assessment points (Palmier-Claus et al., 2011). Out of a possible 720 assessment points, a total of 557 entries were completed by 
participants within 15 minutes of each prompt, representing a $77.4 \%$ overall response rate. Out of a possible 154 days of actigraph monitoring, 150 complete days were recorded (97.4\%). Completion of sleep diaries was also high, as 147 nights out of a possible 154 nights of sleep were subjectively estimated (95.5\%). A full description of sleep and ESM data collected across the study period can be found in Supplementary Table 1.

Do objective / subjective sleep parameters predict next-day daily functioning?

Table two shows that greater objective and subjective SE, subjective SQ and both objective and subjective TST predicted greater next-day functioning scores, while greater objective SF predicted decreased next-day functioning, after adjusting for baseline PANSS scores. 
Does morning NA mediate the relationship between objective / subjective sleep parameters and next-day functioning?

To assess the mediational effect of morning NA on the relationship between sleep and next-day functioning, NA scores at entry point one was included as a mediator variable. All sleep parameters significantly predicted NA upon wakening. When comparing the coefficients between the total (analysis without NA) and direct effects (analysis including NA), NA mediated a varying proportion of the total effects of sleep parameters on next-day functioning (range: $19.5 \%-57.3 \%$ ). Although direct effects of objective and subjective sleep continuity (SE, SF) and quantity (TST) on next-day functioning remained significant, the direct effect of subjective SQ on functioning was not significant (Table 3).

--- INSERT TABLE 3 HERE ---

Do objective / subjective sleep parameters predict next-day psychotic symptoms?

Table four shows that after adjusting for baseline PANSS score, increased objective and subjective SE significantly predicted reduced auditory hallucinations; increased objective SF predicted increased auditory hallucinations and paranoia, while increased subjective SQ predicted reduced auditory hallucinations, paranoia and delusions of control. 
Does morning NA mediate the relationship between objective/subjective sleep parameters and next-day psychotic symptoms?

To assess the mediational effect of morning NA on the relationship between sleep and next-day mean psychotic symptoms, NA scores at entry point one was included as a mediator variable. All sleep parameters significantly predicted NA upon wakening. When comparing the coefficients between the total (analysis without NA) and direct effects (analysis including NA), NA mediated a varying proportion of the total effect of sleep parameters on next-day psychotic symptoms (range: $17.9 \%$ - 50.9\%). Whereas direct effects of objective SF and subjective SQ on paranoia remained significant, direct effects between sleep parameters and both auditory hallucinations and delusions of control failed to reach significance (Table 5).

--- INSERT TABLE 5 HERE ---

\section{Discussion}

This study aimed to determine whether sleep disturbance predicted next-day functioning and psychotic symptoms in people with a diagnosis of schizophrenia. The integration of actigraphy, sleep diaries and ESM enabled the repeated, longitudinal assessment of sleep-wake patterns and ecological assessment of next-day variables, across seven days and nights.

In line with our hypotheses, objective and subjective indices of sleep continuity, quality and duration significantly predicted next-day functioning. Specifically, impairments in all sleep parameters reliably predicted worse functioning after controlling for baseline symptom severity (total PANSS score). For all sleep parameters, morning NA mediated a proportion of 
the total effect on next-day functioning; however, direct effects remained significant for all but one sleep parameter (subjective SQ). This indicates that sleep has a pronounced effect on nextday functioning independent of symptom severity, a proportion of which is mediated by morning NA.

Findings on the relationship between sleep and next-day symptoms also supported our hypotheses. However, only parameters of sleep continuity (SE, SF) and quality (SQ) significantly predicted next-day symptoms. Specifically, reduced subjective and objective SE, reduced subjective SQ and greater objective SF predicted increased auditory hallucinations. Furthermore, greater objective SF and reduced subjective SQ predicted increased paranoia and delusions of control. These results suggest that sleep discontinuity and perceptions of sleep quality are more important than sleep duration, in the prediction of next-day symptoms. For all sleep parameters, morning NA explained a proportion of the effect on next-day symptoms; however, direct effects of objective SF and subjective SQ remained significant for paranoia. In contrast, direct effects between sleep parameters and both auditory hallucinations and delusions of thought control no longer remained significant after including NA as a mediator variable. This suggests that in comparison to auditory hallucinations and delusions of control, the effect of sleep parameters on paranoia may be less mediated by NA.

This study represents the first, well-controlled, longitudinal evaluation of the relationship between objective and subjective sleep-wake patterns, functioning and psychotic symptoms in individuals with a diagnosis of schizophrenia. The findings demonstrate that sleep is a significant predictor of functional and symptomatic outcomes. The uniformity of the results was striking as all parameters predicted functioning and symptoms in the expected direction and some non-significant findings had p-values less than 0.1 , suggesting the analyses might have been underpowered as opposed to there being no effect. Furthermore, the correspondence between objective and subjective sleep parameters in the relationship with 
next-day variables supports the proposition that both may be robust determinants of negative outcomes in people with a diagnosis of schizophrenia.

These findings significantly enhance previous work, which has reported cross-sectional relationships between sleep disturbance and both reduced QoL (Ritnser et al., 2004; Hofstetter et al., 2005) and cognitive functioning (Forest et al., 2007; Keshavan et al., 2004). A key strength of this study was the inclusion of a functioning measure, developed in collaboration with people with lived experience of psychosis. This enabled the examination of how sleep impacts functioning as relevant to those with a diagnosis of schizophrenia. The current findings also converge with previous studies that have reported significant relationships between sleep disturbance and severity of psychotic symptoms (Waters et al., 2011), hallucinations (Reich et al., 1975) and paranoia (Myers et al., 2011). However, our study clearly stands apart for several reasons: 1) it is the first study to employ rigorous, high resolution measurement of sleep parameters and next-day variables over time, within the context of experience sampling methodology, 2) it is the first study to explicitly test mediators of the night-to-day relationship between sleep, individual psychotic symptoms and functioning, 3) it is the first study to integrate actigraphy with ESM measurement on a single device, which may account for our high adherence rates and potentially opens up a novel approach to further investigate sleep and psychosis and 4) our study identifies sleep continuity as an important therapeutic target in the pathway to improved symptom management.

There are several pathways through which objective and subjective sleep disturbance may worsen psychotic symptoms and functioning. This study demonstrated that morning NA mediates a proportion of the total effect of sleep parameters on both functioning and psychotic symptoms. Future studies should extend these results by investigating the mediating role of other potential mechanisms shown to be connected to sleep disturbance and both clinical and functional features of schizophrenia. These could include worry and rumination (Hartley et al., 
2014; Harvey, 2002), hyperactivity of the HPA axis (Buckley \& Shatzberg, 2005), neurocognitive impairment (e.g., Petrovsky et al., 2014), and alterations to dopaminergic function (Volkow et al., 2008).

\section{Limitations}

Despite the novel results described in this study, there are some caveats which require consideration. First, the study relied on the computation of linear associations between variables and as such, no causal inferences can be made regarding any relationships. Second, although psychotic symptoms were assessed, these items were fairly simplistic and may not capture the multidimensional experience of hallucinations and delusions (Haddock et al., 1999). Whether sleep influences the impact or interpretations of such experiences warrants further investigation. Third, the sample included individuals who were chronic in presentation and in receipt of antipsychotic medication. Medication may have confounded the observed relationships between sleep and next-day variables. Future research employing similar methods using antipsychotic naïve patients, or those with early psychosis would validate our findings and permit the investigation of whether sleep predicts symptoms and functioning across the psychosis continuum. Fourth, the use of actigraphy and sleep diaries did not permit investigation of the relationship between sleep architectural parameters or sleep oscillations (e.g. spindles, slow waves) and functioning and symptoms. A rigorous evaluation of these parameters using home-based polysomnography is needed in subsequent studies to localise associations within specific stages or features of sleep. Fifth, two individuals were excluded from the study for exhibiting circadian rhythm disturbances. Future research should explore the effect of other sleep disorders on next-day functioning and symptoms in people with a diagnosis of schizophrenia. Lastly, although levels of alcohol and drug use were notably low 
in our sample, the impact of substance use on sleep parameters and next-day variables could be an interesting avenue for further research.

Concluding remarks

We show that nightly sleep impairment is a significant predictor of poorer next-day functioning and greater symptom severity in individuals with a diagnosis of schizophrenia. In particular, disturbances in sleep continuity and quality, rather than duration are implicated in auditory hallucinations and delusions. These findings suggest that therapeutic interventions targeting sleep may also have the potential to enhance both clinical and functional outcomes for people with schizophrenia (Freeman et al. 2015). Appropriately-powered RCTs of sleep interventions, targeting key parameters identified in the present study, are required to assess the clinical impact on sleep and generalised benefits to symptoms and functioning. 
References

Addington, D., Addington, J., \& Maticka-Tyndale, E. (1993). Assessing depression in schizophrenia: the Calgary Depression Scale. British Journal of Psychiatry, 163(s22), $39-44$.

American Psychiatric Association. (2013). Diagnostic and statistical manual of mental disorders (5th ed.). Arlington, VA: American Psychiatric Publishing.

Bastien, C. H., Vallières, A., \& Morin, C. M. (2001). Validation of the Insomnia Severity Index as an outcome measure for insomnia research. Sleep Medicine, 2(4), 297-307.

Bolger, N., \& Laurenceau, J. P. (2013). Intensive longitudinal methods: An introduction to diary and experience sampling research. Guilford Press.

Buckley, T. M., \& Schatzberg, A. F. (2005). On the interactions of the hypothalamic pituitary adrenal (HPA) axis and sleep: normal HPA axis activity and circadian rhythm, exemplary sleep disorders. The Journal of Clinical Endocrinology \& Metabolism, 90(5), 3106-3114.

Buysse, D. J., Ancoli-lsrael, S., Edinger, J. D., Lichstein, K. L., \& Morin, C. M. (2006). Recommendations for a standard research assessment of insomnia. Sleep, 29(9), 11551173.

Carney, C. E., Buysse, D. J., Ancoli-Israel, S., Edinger, J. D., Krystal, A. D., Lichstein, K. L., \& Morin, C. M. (2012). The consensus sleep diary: standardizing prospective sleep self-monitoring. Sleep, 35(2), 287-302. 
Cassidy, C. M., Schmitz, N., \& Malla, A. (2008). Validation of the alcohol use disorders identification test and the drug abuse screening test in first episode psychosis. Canadian Journal of Psychiatry, 53(1), 26.

Cohrs, S. (2008). Sleep disturbances in patients with schizophrenia. CNS drugs, 22(11), 939962.

Davies, G., Haddock, G., Yung, A. R., Mulligan, L. D., \& Kyle, S. D. (in press). A systematic review of the nature and correlates of sleep disturbance in early psychosis. Sleep Medicine Reviews.

Dawe, S., Seinen, A., \& Kavanagh, D. (2000). An examination of the utility of the AUDIT in people with schizophrenia. Journal of Studies on Alcohol, 61(5), 744-750.

Finan, P. H., Quartana, P. J., \& Smith, M. T. (2015). The effects of sleep continuity disruption on positive mood and sleep architecture in healthy adults. Sleep, 38(11), 1735-1742.

Forest, G., Poulin, J., Daoust, A.-M., Lussier, I., Stip, E., \& Godbout, R. (2007). Attention and non-REM sleep in neuroleptic-naive persons with schizophrenia and control participants. Psychiatry Research, 149(1), 33-40.

Freeman, D., \& Garety, P. A. (2003). Connecting neurosis and psychosis: the direct influence of emotion on delusions and hallucinations. Behaviour research and therapy, 41(8), 923-947.

Freeman, D., Pugh, K., Vorontsova, N., \& Southgate, L. (2009). Insomnia and paranoia. Schizophrenia Research, 108(1), 280-284.

Freeman, D., Waite, F., Startup, H., Myers, E., Lister, R., McInerney, J., ... \& Yu, L. M. (2015). Efficacy of cognitive behavioural therapy for sleep improvement in patients with 
persistent delusions and hallucinations (BEST): a prospective, assessor-blind, randomised controlled pilot trial. The Lancet Psychiatry, 2(11), 975-983.

Haddock, G., McCarron, J., Tarrier, N., \& Faragher, E. B. (1999). Scales to measure dimensions of hallucinations and delusions: the psychotic symptom rating scales (PSYRATS). Psychological Medicine, 29(4), 879-889.

Hartley, S., Haddock, G., Vasconcelos, E. S. D., Emsley, R., \& Barrowclough, C. (2014). An experience sampling study of worry and rumination in psychosis. Psychological Medicine, 44(8), 1605-1614.

Harvey, A. G. (2002). A cognitive model of insomnia. Behaviour Research \& Therapy, 40(8), 869-893.

Harvey, A. G. (2008). Insomnia, psychiatric disorders and the transdiagnostic perspective. Current Directions in Psychological Science, 17(5), 299-303.

Hofstetter, J. R., Lysaker, P. H., \& Mayeda, A. R. (2005). Quality of sleep in patients with schizophrenia is associated with quality of life and coping. BMC Psychiatry, 5(1), 13.

Kawata, A. K., \& Revicki, D. A. (2008). Psychometric properties of the Personal and Social Performance scale (PSP) among individuals with schizophrenia living in the community. Quality of Life Research, 17(10), 1247-1256.

Kay, S. R., Fiszbein, A., \& Opler, L. A. (1987). The positive and negative syndrome scale (PANSS) for schizophrenia. Schizophrenia Bulletin, 13(2), 261.

Kay, S. R., Opler, L. A., \& Lindenmayer, J.-P. (1988). Reliability and validity of the positive and negative syndrome scale for schizophrenics. Psychiatry Research, 23(1), 99-110. 
Keshavan, M. S., Cashmere, J. D., Miewald, J., \& Yeragani, V. K. (2004). Decreased nonlinear complexity and chaos during sleep in first episode schizophrenia: a preliminary report. Schizophrenia Research, 71(2), 263-272.

Kimhy, D., Myin-Germeys, I., Palmier-Claus, J., \& Swendsen, J. (2012). Mobile assessment guide for research in schizophrenia and severe mental disorders. Schizophrenia Bulletin, 38(3), 386-395.

Kupfer, D., Wyatt, R. J., Scott, J., \& Snyder, F. (1970). Sleep disturbance in acute schizophrenic patients. American Journal of Psychiatry, 126(9), 1213-1223.

Manoach, D. S., Demanuele, C., Wamsley, E. J., Vangel, M., Montrose, D. M., Miewald, J., . . Keshavan, M. S. (2014). Sleep spindle deficits in antipsychotic-naïve early course schizophrenia and in non-psychotic first-degree relatives. Frontiers in Human Neuroscience, 8, 762 .

Mooney, C. Z., Duval, R. D., \& Duval, R. (1993). Bootstrapping: A nonparametric approach to statistical inference. Newbury Park, CA: Sage Publications.

Morin, C. M., Belleville, G., Bélanger, L., \& Ivers, H. (2011). The Insomnia Severity Index: psychometric indicators to detect insomnia cases and evaluate treatment response. Sleep, 34(5), 601.

Morosini, P., Magliano, L., Brambilla, L., Ugolini, S., \& Pioli, R. (2000). Development, reliability and acceptability of a new version of the DSM-IV Social and Occupational Functioning Assessment Scale (SOFAS) to assess routine social functioning. Acta Psychiatrica Scandinavica, 101(4), 323-329. 
Myers, E., Startup, H., \& Freeman, D. (2011). Cognitive behavioural treatment of insomnia in individuals with persistent persecutory delusions: a pilot trial. Journal of Behavior Therapy \& Experimental Psychiatry, 42(3), 330-336.

Palmese, L. B., DeGeorge, P. C., Ratliff, J. C., Srihari, V. H., Wexler, B. E., Krystal, A. D., \& Tek, C. (2011). Insomnia is frequent in schizophrenia and associated with night eating and obesity. Schizophrenia Research, 133(1), 238-243.

Palmier-Claus, J., Dunn, G., \& Lewis, S. (2012). Emotional and symptomatic reactivity to stress in individuals at ultra-high risk of developing psychosis. Psychological Medicine, 42(5), 1003-1012.

Palmier-Claus, J. E., Myin-Germeys, I., Barkus, E., Bentley, L., Udachina, A., Delespaul, P. A., . . D Dunn, G. (2011). Experience sampling research in individuals with mental illness: reflections and guidance. Acta Psychiatrica Scandinavica, 123(1), 12-20.

Petrovsky, N., Ettinger, U., Hill, A., Frenzel, L., Meyhöfer, I., Wagner, M., . . K Kumari, V. (2014). Sleep deprivation disrupts prepulse inhibition and induces psychosis-like symptoms in healthy humans. The Journal of Neuroscience, 34(27), 9134-9140.

Pompili, M., Lester, D., Grispini, A., Innamorati, M., Calandro, F., Iliceto, P., . . G Girardi, P. (2009). Completed suicide in schizophrenia: evidence from a case-control study. Psychiatry Research, 167(3), 251-257.

Reeve, S., Sheaves, B., \& Freeman, D. (2015). The role of sleep dysfunction in the occurrence of delusions and hallucinations: A systematic review. Clinical Psychology Review, 42, 96-115.

Reich, L., Weiss, B. L., Coble, P., McPartland, R., \& Kupfer, D. J. (1975). Sleep disturbance in schizophrenia: A revisit. Archives of General Psychiatry, 32(1), 51-55. 
Ritsner, M., Kurs, R., Ponizovsky, A., \& Hadjez, J. (2004). Perceived quality of life in schizophrenia: relationships to sleep quality. Quality of Life Research, 13(4), 783-791.

Saunders, J. B., Aasland, O. G., Babor, T. F., De la Fuente, J. R., \& Grant, M. (1993). Development of the alcohol use disorders identification test (AUDIT). WHO collaborative project on early detection of persons with harmful alcohol consumptionII. Addiction, 88(6), 791-804.

Skinner, H. A. (1982). The drug abuse screening test. Addictive Behaviors, 7(4), 363-371.

Tahmasian, M., Khazaie, H., Golshani, S., \& Avis, K. T. (2013). Clinical application of actigraphy in psychotic disorders: a systematic review. Current Psychiatry Reports, $15(6), 1-15$.

Volkow, N. D., Wang, G.-J., Telang, F., Fowler, J. S., Logan, J., Wong, C., . . Thanos, P. K. (2008). Sleep deprivation decreases binding of [11C] raclopride to dopamine D2/D3 receptors in the human brain. The Journal of Neuroscience, 28(34), 8454-8461.

Waters, F., Allen, P., Aleman, A., Fernyhough, C., Woodward, T. S., Badcock, J. C., ... \& Vercammen, A. (2012). Auditory hallucinations in schizophrenia and nonschizophrenia populations: a review and integrated model of cognitive mechanisms. Schizophrenia Bulletin, 38(4), 683-693.

Waters, F., Sinclair, C., Rock, D., Jablensky, A., Foster, R. G., \& Wulff, K. (2011). Daily variations in sleep-wake patterns and severity of psychopathology: A pilot study in community-dwelling individuals with chronic schizophrenia. Psychiatry Research, 187(1), 304-306.

Wilson, S. J., Nutt, D. J., Alford, C., Argyropoulos, S. V., Baldwin, D. S., Bateson, A. N., ... \& Wade, A. G. (2010). British Association for Psychopharmacology consensus 
statement on evidence-based treatment of insomnia, parasomnias and circadian rhythm disorders. Journal of Psychopharmacology, 24(11), 1577-1601.

Wood, L., Price, J., Morrison, A., \& Haddock, G. (2013). Exploring service users perceptions of recovery from psychosis: A Q-methodological approach. Psychology \& Psychotherapy: Theory, Research \& Practice, 86(3), 245-261.

World Health Organization. (1992). The ICD-10 classification of mental and behavioural disorders: clinical descriptions and diagnostic guidelines. Geneva: World Health Organization.

Xiang, Y.-T., Weng, Y.-Z., Leung, C.-M., Tang, W.-K., Lai, K. Y., \& Ungvari, G. S. (2009). Prevalence and correlates of insomnia and its impact on quality of life in Chinese schizophrenia patients. Sleep, 32(1), 105.

Yang, M., Morin, C. M., Schaefer, K., \& Wallenstein, G. V. (2009). Interpreting score differences in the Insomnia Severity Index: using health-related outcomes to define the minimally important difference. Current Medical Research \& Opinion, 25(10), 24872494. 